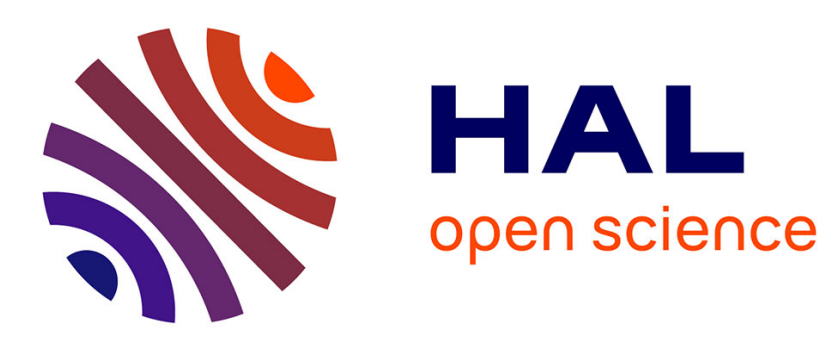

\title{
Nonlinear Microwave Phenomena in Ferrite Films : Basic Physics and Applications (Invited)
}

\author{
A. Slavin
}

\section{To cite this version:}

A. Slavin. Nonlinear Microwave Phenomena in Ferrite Films: Basic Physics and Applications (Invited). Journal de Physique IV Proceedings, 1997, 07 (C1), pp.C1-379-C1-382. 10.1051/jp4:19971155 . jpa-00254800

\section{HAL Id: jpa-00254800 https://hal.science/jpa-00254800}

Submitted on 1 Jan 1997

HAL is a multi-disciplinary open access archive for the deposit and dissemination of scientific research documents, whether they are published or not. The documents may come from teaching and research institutions in France or abroad, or from public or private research centers.
L'archive ouverte pluridisciplinaire HAL, est destinée au dépôt et à la diffusion de documents scientifiques de niveau recherche, publiés ou non, émanant des établissements d'enseignement et de recherche français ou étrangers, des laboratoires publics ou privés. 


\title{
Nonlinear Microwave Phenomena in Ferrite Films: Basic Physics and Applications (Invited)
}

\author{
A.N. Slavin \\ Physics Department, Oakland University, Rochester, MI 48309, U.S.A.
}

\begin{abstract}
A short overview of the theory and applications of noninear spin wave phenomena in ferrite films at microwave frequencies is given. A method of classical Hamiltonian formalism for spin waves in bulk ferrimagnets in combination with the theory of spin wave spectrum for thin magnetic films is used for theoretical description of nonlinear spin wave phenomena in films. Comparative roles of three-wave and four-wave interaction processes in the nonlinear dynamics of spin waves in films are discussed. A local character of spin wave exciration by the magnetic field of a strip-line antenna is taken into account. Several examples of nonlinear microwave signal processing devices based on the nonlinear dynamics of spin waves in ferrite films (frequency-selective limiter, signal-to-noise enhancer, convolver, and a soliton delay line) are presented.
\end{abstract}

\section{INTRODUCTION}

Spin waves (SW) at microwave frequencies propagating in monocrystalline ferrite films provide a superb opportunity to study nonlinear wave phenomena in solids. Nonlinear and dispersive characteristics of $S W$ in ferrite films can be controlled by changing the magnitude and orientation of the bias magnetic field, and a wide variety of nonlinear wave phenomena (like parametric and kinetic instabilities of SW, auto-oscillations, chaotic dynamics, strange attractors, formation, propagation, and collision of "bright " and "dark" SW envelope solitons) can be observed in ferrite films at moderate input microwave power levels of less than $1 \mathrm{~W}$ (see e.g. $[1,2]$ ). An additional advantage of ferrite films as a medium for experimental investigations of nonlinear wave dynamics is that the wave process in the film is directly accessible from the surface for characterization by either inductive probes, or magnetooptical methods ( see Chapter 1 in [2] and references therein).

Nonlinear dynamics of $\mathrm{SW}$ in ferrite films can be described theoretically by the classical Hamiltonian formalism for SW in unbounded ferromagnet [3] in combination with the dipole-exchange theory of SW spectrum in magnetic films (see [4], and Chapter 2 in [2]). A brief outline of this theory is given in the section 2 of this paper.

Processes of nonlinear SW interaction in ferrite films are also perspective for applications in satellite communication and radar technology. These processes can be used to design a new generation of nonlinear SW signal processing devices that are magnetically tunable in a wide frequency range, and could be easily integrated in the modern planar technology. In particular, such devices include frequency selective limiters [5], signal-to-noise enhancers [5], non-resonant and resonant convolvers $[6,7]$, and recently developed active soliton delay lines and soliton devices for pulse shaping and compression [8]. A brief description of some of these devices is given in section 3 of this paper.

\section{HAMILTONIAN FORMALISM FOR SPIN WAVES IN FERRITE FILMS}

The classical Hamiltonian formalism for spin waves in bulk ferromagnets has been developed by V.E. Zakharov, V.S. L'vov, and S.S. Starobinets (see e.g. [3]). This theory has been extended for the case of thin ferrite films in [4, 9] where discreteness of the spin wave spectrum in films has been taken into account. In the case of a film of a finite thickness variable magnetization $\mathbf{m}(\xi, \boldsymbol{r})$ in the film is expanded in a series of inhomogeneous plane waves that are propagating in a film plane along the direction of a wave vector $\mathbf{k}$, and have certain distributions of magnetization amplitude along the film thickness ( $\xi$-direction) described by the functions $\Phi_{n}(\xi)$ (spin wave modes) that satisfy the exchange boundary conditions at the film boundaries This expansion has the form $[4,9]$ :

$$
\mathbf{m}(\xi, \mathbf{r})=\sum_{n, \mathrm{k}} \mathbf{m}_{n \mathbf{k}} \Phi_{n}(\xi) \exp (\mathbf{i k r})
$$

The energy of the film, which includes the energies of the Zeeman interaction with external magnetic fields ( constant bias magnetic field and the time-dependent field of external excitation signal), dipole-dipole, and exchange interactions is written in terins of vector amplitudes of spin wave modes $\mathbf{m}_{n \mathbf{k}}$. After transformation to a new set of variables $b_{n \mathbf{k}}, b^{*}{ }_{n \mathbf{k}}$ (HolsteinPrimakoff transformations [3]) equations of motion assume a standard Hamiltonian form, and the energy of the film becomes a Hamiltonian function $[3,4,9]$. One of the major assumptions in this approach is that the amplitude of the variable magnetization $m$ is small compared to the saturation magnetization in the medium $M_{0}$, and, therefore, the transformed 
amplitudes of spin wave modes $b_{n \mathrm{k}}$ (that are proportional to $\sqrt{m^{2} / 2 M_{0}^{2}}$ ) are also small. This assumption allows us to expand the spin wave Hamiltonian function of the film in a Taylor series in powers of the spin wave amplitudes $b_{n \mathbf{k}}$. The spin wave Hamiltonian function of the film then takes the form:

$$
\mathscr{H}\left(b_{n \mathbf{k}}, b_{n \mathbf{k}}^{*}\right)=\mathscr{H}^{(2)}+\mathscr{H}^{(3)}+\mathscr{H}^{(4)}+\ldots+\mathcal{H}_{p}^{(1)}+\mathscr{H}_{p}^{(2)}+\ldots .
$$

where $\Re^{(2)}$ describes the "kinetic energy" of spin waves

$$
\mathscr{H}^{(2)}\left(b_{n \mathbf{k}}, b_{n \mathbf{k}}^{*}\right)=\sum_{n} \int \omega_{n \mathbf{k}}(\mathbf{k}) b_{n \mathbf{k}} b_{n \mathbf{k}}^{*} d \mathbf{k},
$$

and $\omega_{n \mathbf{k}}(\mathbf{k})$ is the dispersion relation describing the discrete spin wave spectrum in a film ( $\operatorname{see}[4,9]$ ). The terms $\Re_{p}^{(1)}$ and $\dddot{H}^{(2)} p$, originated from the Zeeman interaction and containing one and two spin wave amplitudes correspondingly, describe linear excitation of spin waves by variable magnetic field components perpendicular to the saturation magnetization $\boldsymbol{M}_{\boldsymbol{\theta}}$, and parametric excitation of spin wave pairs by the variable field component parallel to $\boldsymbol{M}_{0}$. The terms $\Re^{(3)}$ and $\Re^{(4)}$ describe nonlinear interaction processes in the system of spin waves. The three-wave terms $\Re^{(3)}$ originated from the dipole-dipole interaction describe spin wave interaction processes with conservation laws:

$$
\omega_{1}\left(\mathbf{k}_{1}\right)=\omega_{2}\left(\mathbf{k}_{2}\right)+\omega_{3}\left(\mathbf{k}_{3}\right), \quad \mathbf{k}_{1}=\mathbf{k}_{2}+\mathbf{k}_{3} .
$$

These processes are analogous to the first-order Suhl processes [10] and have the lowest power threshold

$$
b_{1 t h}^{2}=\eta^{2} / V_{n k}^{2}
$$

where $\eta=\gamma \Delta \mathrm{H}_{\mathrm{x}}$ is the relaxation parameter and $V_{n k}$ is the matrix element of three-wave interaction defined in [4]. If the pumping signal $\left(\omega=\omega_{1}\right)$ is localized in the region of the width $W$, threshold of these processes is higher (see p.64 in [2]):

$$
b_{1 t h}^{2}=\frac{\eta^{2}+\left(\beta \pi V_{g} / W\right)^{2}}{V_{n k}^{2}},
$$

where $\beta$ is the dimensionless coefficient depending on the geometry of the pumping localization and $V_{\mathrm{g}}$ is the group velocity of parametrically excited waves. Low threshold three-wave processes can, however, be prohibited by conservation laws (e.g. when $\left.\omega_{1} / 2<\omega_{n k}(0)\right)$. In that case the spin wave nonlinearity is determined by the four-wave interaction processes described by the term $\Re^{(4)}$, and having the conservation laws:

$$
\omega_{1}\left(\mathbf{k}_{1}\right)+\omega_{2}\left(\mathbf{k}_{2}\right)=\omega_{3}\left(\mathbf{k}_{3}\right)+\omega_{4}\left(\mathbf{k}_{4}\right), \quad \mathbf{k}_{1}+\mathbf{k}_{2}=\mathbf{k}_{3}+\mathbf{k}_{4} .
$$

These processes are analogous to the second-order Suhl processes [10] and are always allowed. Their threshold is given by :

$$
b_{2 t h}^{2}=\eta / T_{n k}
$$

where $T_{n k}$ is the matrix element of four-wave interaction defined in [4]. The threshold of four-wave processes (8) is substantially higher then the threshold of three-wave processes under homogeneous pumping (5), but can be of comparable to the threshold of three-wave processes under localized pumping (6). The competition between these two types of nonlinear processes determines nonlinear properties of SW in ferrite films in most experimental cases. The performance of the nonlinear microwave signal processing devices described below is also based on three-wave and four-wave processes of SW interaction.

\section{NONLINEAR SPIN-WAVE DEVICES FOR MICROWAVE SIGNAL PROCESSING}

The review of operational characteristics of some of the nonlinear signal processing devices based on the properties of dipolar microwave SW (or magnetostatic waves (MSW)) propagating in thin ferrite films are presented in [5]. We describe below four examples of such devices: power limiter, signal-to-noise enhancer (SNE), convolver, and a soliton transmission line.

The operation of the power limiter and SNE is based on the three-wave decay process (4), while the operation of the resonant convolver is based on the three-wave confluence process also described by (4). The operation of the soliton transmission line, based on the four-wave process (7) of modulational instability $\left(k_{1}=k_{2}=k_{0}, k_{3,4}=k_{0} \pm \kappa, \kappa<<k_{0}\right)$, is only possible in the spectral region where low threshold three-wave processes (4) are prohibited. 
Fig. 1 shows the basic configuration of a nonlinear MSW transmission line which may be used as a microwave power limiter [5]. It consists of a thin yttrium-iron garnet (YIG) film (typical thickness 5-100 $\mu \mathrm{m}$, dimensions $2 \mathrm{~mm} \times 10 \mathrm{~mm}$ ) grown on nonmagnetic gallium-gadolinium-garnet (GGG) substrate (thickness $0.5 \mathrm{~mm}$ ). The film is placed on top of the alumina substrate. Two microstrip transducers (typical width $50 \mu \mathrm{m}$, length $2 \mathrm{~mm}$ ) are deposited on the surface of the substrate. The structure is placed in external DC magnetizing field $H$. The field of the strength $H=0.1-3 \mathrm{kOe}$ is created by an electromagnet or by a miniature permanent magnetic system. Input EM signal fed to the input transducer excites a surface MSW which propagates in the film. The output transducer converts the MSW back into the EM signal. Fig. 2 shows typical dependence of output power versus input power for the device at the $3 \mathrm{GHz}$ frequency. Due to nonlinear decay (4) of a surface MSW (of frequency $\omega_{1}$ ) into two backward volume MSW (of frequency $\omega_{2} \cong \omega_{3} \cong \omega_{1} / 2$ ) the power of the output signal (at $\omega_{1}$ ) remains nearly constant with variation of input signal power in the wide dynamic range (around $20 \mathrm{~dB}$ ).

Operational frequency of the limiter and level of limitation can be changed within $0.5-5 \mathrm{GHz}$ frequency band and $0.1-100 \mathrm{~mW}$ power range respectively, by adjusting parameters of the YIG film structure and DC magnetizing field.

The above described limiter has an additional advantage of being frequency selective i.e. it is capable of limiting simultaneously several microwave signals of different frequencies without cross-interference if the frequency separation between the signals is of the order of several $\mathrm{MHz}$ [5].

The MSW signal-to-noise enhancer (SNE) allows to increase signal to noise ratio for a microwave signal. Fig. 3 shows the schematic view and explains principle of the SNE operation [5]. It contains a YIG film, alumina substrate, and two (input and output) $50-\Omega$ lines connected to a narrow (typically 20-100 $\mu \mathrm{m}$ ) microstrip transducer.

When a low power input EM signal (typical power less than $1 \mathrm{~mW}$ ) is fed to the input line, the microstrip transducer excites surface MSW that propagate away from the transducer, carry energy from the transducer, and are absorbed by the absorbers. Only a small part of the microwave power passes to the output line and device provides high transmission loss for the low power signal. When a high power EM signal (typical power above $100 \mathrm{~mW}$ ) is fed to the input line, the process of the MSW excitation is saturated (see Fig.2) and almost all the power goes to the output line. Thus, SNE provides low transmission loss for high power signal. Fig. 4. shows typical dependence of transmission loss versus input power for a SNE. The carrier frequency of the input signal may be adjusted in the $1-5 \mathrm{GHz}$ frequency band. The SNE allows to improve signal-to-noise ratio for both continuous and pulsed (pulse duration larger than several $\mu \mathrm{s}$ ) microwave signals by a factor of 10-20 dB. SNE similar to the described above, but based on a balance mixer principle, has been developed recently by Murata MFG Co. (Japan) for applications in a satellite TV receiving systems [11].

MSW convolvers allow to perform convolution of two short radio pulses directly at microwave frequencies. A schematic view of an MSW convolver is shown in Fig. 5. It consists of an alumina substrate with three microstrip transducers deposited on its surface and a YIG film pressed to the transducers. The structure is placed in the external DC tangential magnetizing field. Two transducers are used to excite MSW pulses $h\left(\omega_{2}, t\right)$ and $g\left(\omega_{3}, t\right)$ propagating in the YIG film. Three-wave confluence (4) of the excited wave pulses results in the formation of the output signal $q\left(\omega_{1}, t\right)$ (received by the third transducer (see Fig. 6)) which is proportional to the convolution of the input signals $q(t) \sim \int g(t) h(t-\tau) d \tau$. There are two types of MSW convolvers.

MSW convolvers of a non-resonance type [6] are based on the confluence of two contra-propagating backward volume waves into a uniform precession signal with double frequency. Operating frequency of these convolvers lies in the $1-10 \mathrm{GHz}$ frequency band. They have instantaneous frequency band width of up to $500 \mathrm{MHz}$ and internal bi-linearity coefficient (measure of convolution efficiency) of $-10 . . .-14 \mathrm{dBm}$.

MSW convolvers of a resonance type [7] use confluence of two backward volume waves (frequencies $\omega_{2}$ and $\omega_{3}$ ) propagating in opposite directions (or at the small angle to each other) which results in generation of a third volume or surface MSW of the frequency $\omega_{1}=\omega_{2}+\omega_{3}$. Operating frequency of these convolvers lies in the 1-2 GHz frequency band, instantaneous bandwidth is equal to $150 \mathrm{MHz}$. Convolvers of this type have very high internal bi-linearity coefficient $-2 \ldots-12 \mathrm{dBm}$. MSW convolvers may be used in microwave radar and communications systems instead of the surface acoustic wave (SAW) convolvers, which are used now for processing of radio pulses with carrier frequencies in the 0.1-0.5 GHz frequency range.

The MSW soliton transmission line has the same configuration as the microwave power limiter shown in Fig. 1, but the bias magnetic field and the carrier frequency of the input pulsed signal are chosen in such away that the three-wave processes (4) are prohibited, and the conditions for formation of MSW envelope solitons are satisfied (see chapter 9 in [1]). This transmission line may be used to compress short microwave pulses when MSW [8]. Compression of microwave pulses with carrier frequency in the range 3-10 GHz, duration $10-40 \mathrm{~ns}$, and peak power 1-2 W to the pulses with duration of $2-5 \mathrm{~ns}$ has been realized. Fig. 7 demonstrates the shape of input microwave pulse (duration $10 \mathrm{~ns}$, carrier frequency $4.5 \mathrm{GHz}$, peak power $1 \mathrm{~W}$ ) and the shape of compressed microwave pulse (duration $2.5 \mathrm{~ns}$ ) at the MSW soliton transmission line output.

Nonlinear compression of MSW packets allows to eliminate dispersion spreading of microwave pulses in the MSW delay line. This property may be used to design active microwave delay lines containing the MSW soliton delay line and a microwave amplifier connected in the feedback loop. These active delay lines are expected to provide delay times of up to 10-20 $\mu$ sith insertion loss of $10 \mathrm{~dB} / \mu$ s directly at microwave frequencies [8].

\section{Acknowledgments}

It is a pleasure to thank Prof. Yu.K. Fetisov for preparation of the material on the nonlinear spin wave devices. This work was partially supported by the Oakland University Foundation and by the Research Corporation (Grant CC-3401). 


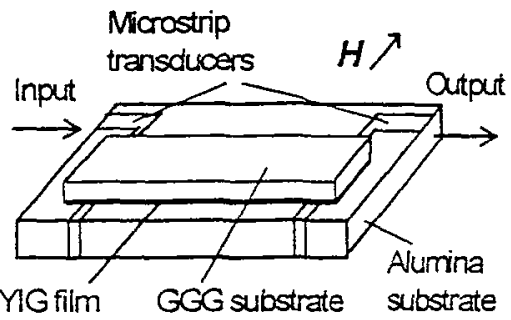

Fig. I MSW transmission line

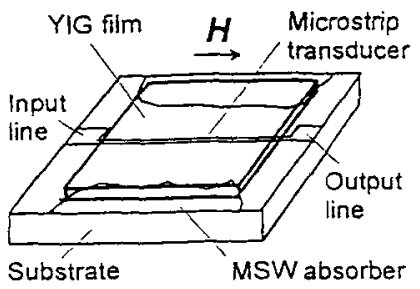

Fig. 3 MSW signal-to-noise enhancer (SNE).

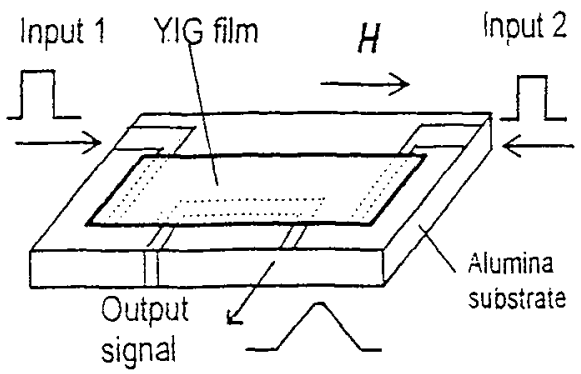

Fig. 5 MSW convolver.

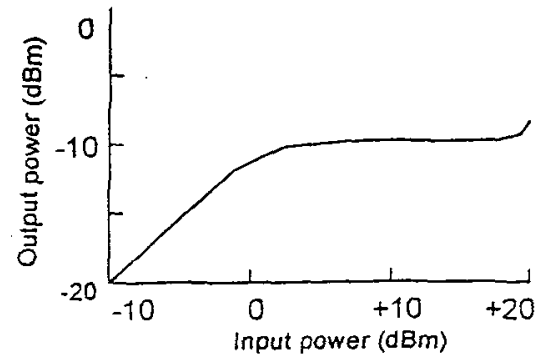

Fig. 2 Characteristic of an MSSW limiter.

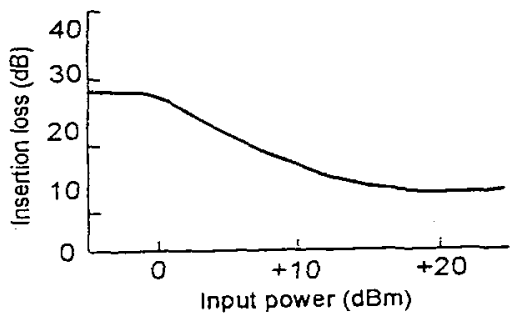

Fig. 4 Characteristic of an MSW SNE.

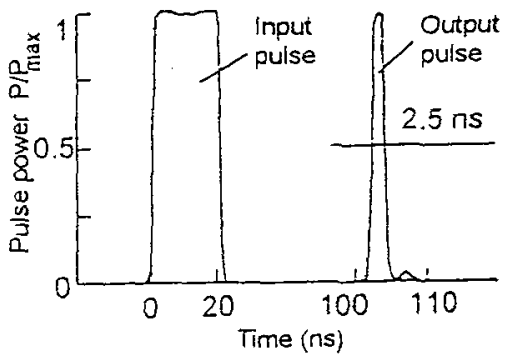

Fig. 6 Compression of EM pulses in MSW soliton transmission line.

\section{References}

[1]. Wigen P.E. , ed., Nonlinear Phenomena and Chaos in Magnetic Materials (World Scientific, Singapore, 1994).

[2]. Cottam M.G., ed. , Linear and Nonlinear Spin Waves in Magnetic Films (World Scientific, Singapore, 1994).

[3]. L'vov V.S., Wave Turbulence under Parametric Excitation (Springer, Berlin, 1994) pp. 27-34.

[4]. Slavin A.N. and Rojdestvenski I.V., IEEE Trans.Magn. 30 (1994) 37-50.

[5]. Stitzer S.N, and Emtage P.R., Circuits, Systems, and Signal Processing 4 (1985) 227-252.

[6]. Parekh J. P., Tuan H. S. and Chang K. W., IEEE Trans. on Sonics and Ultrusonics, 32 (1985) 691-698.

[7]. Kovshikov N. G., Kolodin P. A., and Kostylev M. P., Proceedings of the 22-th European Microwave Conference, Espoo, Finland (1992) 257-260.

[8]. Fetisov Y.K., Kabos P. and Patton C. E., Proceedings of the INTERMAG-96 Conference, Seattle, USA (1996), to be published in IEEE Trans.Magn. .

[9]. Slavin A.N. and Kalinikos B.A., Sov.Phys.Tech.Phys. 32 (1987) 1446-48.

[10]. Suhl H., J. Phys. Chem. Solids, 1 (1957) 209.

[11]. Nomoto T., IEEE Trans. MTT-41 (1993) 1316.Ishak W.S., Proceedings of IEEE, 76 (1988) 171 179. 